Commun. math. Phys. 4, 137-142 (1967)

\title{
Über Lösungen der Einsteinschen Feldgleichungen, die sich in einen fünfdimensionalen flachen Raum einbetten lassen
}

\author{
H. STEPHANI \\ Theoretisch-Physikalisches Institut der Universität Jena*
}

Eingegangen am 15. Oktober 1966

\begin{abstract}
Solutions of the Einstein equations which can be embedded in a flat five dimensional space are studied, without referring to the explicit form of the embedding. Those describing the gravitational field of a perfect fluid or an electromagnetic null field are invariantly characterized; in some special cases the metric is fully determined.

Nach einer kurzen Darstellung des Lösungsweges werden diejenigen Lösungen der Einsteinschen Feldgleichungen invariant charakterisiert und zum Teil vollständig angegeben, die einer idealen Flüssigkeit oder einem elektromagnetischen Nullfeld entsprechen und sich in einen fünfdimensionalen flachen Raum einbetten lassen; auf die explizite Form der Einbettung wird dabei kein Bezug genommen.
\end{abstract}

\section{Einführung}

Das Problem, einen Riemannschen Raum in einen quasieuklidischen Raum einzubetten, ist mehr mathematischer als physikalischer Natur und wurde deshalb im Zusammenhang mit Lösungen der Einsteingleichungen früher selten untersucht. In der letzten Zeit jedoch erschien eine Reihe von Arbeiten, die sich mit diesem Problem befassen [1] - [7].

In der vorliegenden Arbeit wird versucht, alle Lösungen, die das Gravitationsfeld einer idealen Flüssigkeit oder eines elektromagnetischen Nullfeldes beschreiben und sich in einen fünfdimensionalen flachen Raum einbetten lassen, vollständig zu bestimmen oder wenigstens invariant zu charakterisieren. Die explizite Form der Einbettung interessiert dabei nicht.

Als Gegenstück zur Petrov-Klassifizierung könnte man die Lösungen der Feldgleichungen auch nach der zur Einbettung benötigten Dimensionszahl klassifizieren und ähnlich wie dort versuchen, die „einfachen” Typen, hier also die in fünf, sechs oder sieben Dimensionen einbettbaren, vollständig anzugeben; die in dieser Arbeit durchgeführten Unter-

\footnotetext{
* Adresse: 69 Jena, Max-Wien-Platz 1, DDR.
} 
suchungen wären der erste Teil eines solchen Programms. Die Schwierigkeiten wachsen mit der Dimensionszahl jedoch sehr stark an, so daß es zweifelhaft ist, ob in ähnlicher Weise auch Räume höherer Klasse behandelt werden können.

Ein Teil der Ergebnisse über Gravitationsfelder idealer Flüssigkeiten ist schon in der Untersuchung von SzEKERES [7] enthalten, von der der Verfasser während der Abfassung des Manuskriptes Kenntnis erhielt.

\section{Problemstellung und Skizzierung des Lösungsweges}

Der vierdimensionale Riemannsche Raum werde durch einen metrischen Tensor $g_{i k}$ der Signatur $(+++-)$ beschrieben, aus dem der Krümmungstensor und seine Verjüngung gemäß

$$
a_{i ; j ; k}-a_{i ; k ; j}=a^{n} R_{n i j k}, \quad R_{i k} \equiv R_{i n k}^{n}
$$

gebildet werden. Dieser Raum kann bekanntlich [8] genau dann in einen fünfdimensionalen flachen Raum der Signatur $(+++-e), e= \pm 1$, eingebettet werden, wenn ein symmetrischer Tensor $\Omega_{i \hbar}$ existiert, der den Gleichungen

$$
\begin{gathered}
R_{i j k l}=e\left(\Omega_{i k} \Omega_{j l}-\Omega_{i l} \Omega_{j k}\right) \quad(\mathrm{GAUSS}) \\
\Omega_{i k ; j}=\Omega_{i j ; k} \quad(\text { CoDAZzI })
\end{gathered}
$$

genügt. Außerdem müssen noch die Einsteinschen Feldgleichungen erfüllt sein, die wegen (2) zu

$$
\Omega_{i k} \Omega_{n}^{n}-\Omega_{i n} \Omega_{k}^{n}-\frac{1}{2} g_{i k}\left(\Omega_{n}^{n} \Omega_{m}^{m}-\Omega_{m n} \Omega^{m n}\right)=e T_{i k}
$$

äquivalent sind.

Um nun Lösungen zu bestimmen, die diesen Gleichungen genügen, geben wir uns zuerst einen Energieimpulstensor $T_{i k}$ eines einfachen Typs vor; wir wählen den der idealen Flüssigkeit

$$
T_{i k}=(\mu+p) u_{i} u_{k}+p g_{i k}, \quad u_{i} u^{i}=-1 \text {, }
$$

mit der Vierergeschwindigkeit $u_{i}$, der Ruhemassendichte $\mu$ und dem Druck $p$ oder den eines elektromagnetischen Nullfeldes

$$
T_{i k}=\lambda k_{i} k_{k}, \quad k_{i} k^{i}=0 .
$$

Dann ergänzen wir die hier auftretenden Vektoren zu einem Vierbein und entwickeln den Tensor $\Omega_{i k}$ nach diesem Vierbein. Die Basisvektoren werden so gewählt, daß $\Omega_{i k}$ möglichst einfach wird; z. B. nehmen wir die Richtung von $\Omega_{i k} u^{i}+A u_{k}, A=\Omega_{i k} u^{i} u^{k}$, als $v$-Richtung usw. Wir 
machen also die Ansätze

$$
\begin{aligned}
\Omega_{i k}= & A u_{i} u_{k}+B\left(u_{i} v_{k}+v_{i} u_{k}\right)+C v_{i} v_{k}+D\left(v_{i} w_{k}+v_{k} w_{i}\right)+ \\
& +E w_{i} w_{k}+F\left(w_{i} z_{k}+z_{i} w_{k}\right)+G z_{i} z_{k} \\
g_{i k}= & -u_{i} u_{k}+v_{i} v_{k}+w_{i} w_{k}+z_{i} z_{k} \\
\Omega_{i k}= & A m_{i} m_{k}+C\left(m_{i} k_{k}+k_{i} m_{k}\right)+D k_{i} k_{k}+E\left(w_{i} k_{k}+k_{i} w_{k}\right)+ \\
& +F w_{i} w_{k}+G\left(w_{i} z_{k}+z_{i} w_{k}\right)+H z_{i} z_{k} \\
g_{i k}= & m_{i} k_{k}+k_{i} m_{k}+w_{i} w_{k}+z_{i} z_{k} \\
\Omega_{i k}= & B\left(m_{i} w_{k}+m_{k} w_{i}\right)+C\left(m_{i} k_{k}+m_{k} k_{i}\right)+E\left(k_{i} w_{k}+w_{i} k_{k}\right)+ \\
& +F w_{i} w_{k}+D k_{i} k_{k}+K\left(k_{i} z_{k}+z_{i} k_{k}\right)+H z_{i} z_{k} \\
g_{i k}= & m_{i} k_{k}+k_{i} m_{k}+w_{i} w_{k}+z_{i} z_{k}
\end{aligned}
$$

mit

$$
-u^{2}=v^{2}=w^{2}=z^{2}=1 \quad \text { bzw. } \quad m k=w^{2}=z^{2}=1,
$$

alle anderen Skalarprodukte 0.

Mit diesen Ausdrücken gehen wir in (4) ein und erhalten die folgenden möglichen Tensoren $\Omega_{n m}$ :

$$
\begin{aligned}
& \Omega_{n m}=C g_{n m}+D u_{n} u_{m} \quad \text { ideale Flüssigkeit, Fall } 1 \\
& \Omega_{n m}=C g_{n m}+2 C u_{n} u_{m}+D v_{n} v_{m} \text { ideale Flüssigkeit, Fall } 2 \\
& \Omega_{n m}=k_{n} k_{m}+H z_{n} z_{m} \quad \text { Strahlungsfeld, Fall } 1 \\
& \Omega_{n m}=k_{n} z_{m}+z_{n} k_{m} \quad \text { Strahlungsfeld, Fall } 2 \text {. }
\end{aligned}
$$

Im nächsten Schritt befriedigen wir die Codazzi-Gleichungen (3), indem wir die Ableitungen der Basisvektoren und der Koeffizienten wieder nach den Basisvektoren entwickeln. Die damit gewonnenen Aussagen (Hyperflächennormalität der benutzten Basisvektoren usw.) benutzen wir zur Aufstellung eines geeigneten einfachen Koordinatensystems, in dem wir dann die Gauß-Gleichungen (2) lösen.

Die hierbei durchzuführenden Rechnungen sind zum Teil ziemlich umfangreich. Wir werden uns deshalb darauf beschränken, die Ergebnisse darzustellen.

\section{Ideale Flüssigkeit - Fall 1}

Dieser Typ ist durch folgende Strukturen gekennzeichnet:

$$
\begin{aligned}
\Omega_{i k}= & C g_{i k}+D u_{i} u_{k} \\
T_{i k}= & 3 C^{2} u_{i} u_{k}+\left(2 D C-3 C^{2}\right)\left(v_{i} v_{k}+w_{i} w_{k}+z_{i} z_{k}\right) \\
R_{i j k l}= & C^{2}\left(g_{i k} g_{j e}-g_{i e} g_{j k}\right)+ \\
& +C D\left(g_{i k} u_{j} u_{e}+u_{i} u_{k} g_{j e}-g_{i e} u_{j} u_{k}-u_{i} u_{e} g_{j k}\right) .
\end{aligned}
$$


Da die Ruhemassendichte positiv sein muß, kommt nur eine Einbettung mit $e=+1$ in Frage.

Der Konformtensor ist null, die Räume sind konform flach.

Als Spezialfall $D=0$ sind die Räume konstanter Krümmung enthalten, mit $T_{i k}=-3 C^{2} g_{i k}$.

Aus den Codazzi-Gleichungen folgt, daß die Strömung rotationsund scherungsfrei (vgl. [9]) ist.

Wenn auch die Expansion verschwindet, so ist der zur Vierergeschwindigkeit senkrechte Unterraum ein Raum konstanter Krümmung, und die allgemeine Lösung

$$
\begin{aligned}
d s^{2} & =\frac{d r^{2}}{1-C^{2} r^{2}}+r^{2}\left(\sin \vartheta^{2} d \varphi^{2}+d \vartheta^{2}\right)-u_{4}^{2} d t^{2} \\
u_{4} & =r \beta(t) \sin \vartheta \sin [\varphi-\delta(t)]+r \gamma(t) \cos \vartheta+\tau(t) \sqrt{1-C^{2} r^{2}}+g(t) \\
\mu & =3 C^{2}=\text { const. } \\
p & =-\mu-\frac{2}{3} \mu g(t) u^{4}
\end{aligned}
$$

ist vom Typ der inneren Schwarzschildlösung (die als Spezialfall enthalten ist), mit konstanter Ruhemassendichte; $\gamma, \beta, \delta, \tau$ und $g$ sind beliebige Zeitfunktionen.

Ist die Expansion $\alpha$ von null verschieden, so ist der Ortsraum ein Raum konstanter, aber zeitabhängiger Krümmung. Nehmen wir zusätzlich an, daß sich die Metrik des Ortsraumes in der Gestalt

$$
d^{(3)} s^{2}=\left(d x^{2}+d y^{2}+d z^{2}\right) V^{-2}
$$

darstellen läßt, so erhalten wir als Lösungen

$$
\begin{aligned}
d s^{2} & =\frac{d x^{2}+d y^{2}+d z^{2}}{V^{2}}-\left(\frac{V, 4}{\alpha V}\right)^{2} d t^{2} \\
V & =a+\frac{C^{2}-\alpha^{2}}{4}\left[\left(x-x_{0}\right)^{2}+\left(y-y_{0}\right)^{2}+\left(z-z_{0}\right)^{2}\right] \\
\mu & =3 C^{2} \quad p=-\mu+2 C C,_{4} \frac{V}{V,,_{4}}
\end{aligned}
$$

mit beliebigen Zeitfunktionen $C, a, \alpha, x_{0}, y_{0}$ und $z_{0}$. In dieser Lösungsmannigfaltigkeit sind alle einbettbaren Lösungen mit inkohärenter Materie enthalten; dies sind genau die Friedmannmodelle.

\section{Ideale Flüssigkeit - Fall 2}

Diese Räume werden beschrieben durch

$$
\begin{aligned}
& \Omega_{i k}=2 C u_{i} u_{k}+C g_{i k}+D v_{i} v_{k} \\
& T_{i k}=C(3 C+2 D) u_{i} u_{k}+C^{2}\left(v_{i} v_{k}+w_{i} w_{k}+z_{i} z_{k}\right),
\end{aligned}
$$

sie sind vom Typ $I_{e}$. 
Es gibt keine Lösung mit $2 C+D=0$, also keine mit einem Energieimpulsator proportional zu $g_{i k}$.

Die Vektoren $u$ und $v$ (Vierergeschwindigkeit und Viererbeschleunigung) sind hyperflächennormal, und es läßt sich ein zweifach orthogonales Koordinatensystem einführen. Es gibt keine beschleunigungsfreien Lösungen. Spezialfälle der Räume mit nichtverschwindender Viererbeschleunigung sind

$$
\begin{gathered}
d s^{2}=r^{2} d \Omega+\frac{\varepsilon\left(K+2 k r^{2}\right)}{K+k r^{2}}-\left(K+k r^{2}\right) d t^{2} \\
d \Omega= \begin{cases}d \vartheta^{2}+\sin ^{2} \vartheta d \varphi^{2} & \varepsilon=+1 \\
d \vartheta^{2}+\sinh ^{2} \vartheta d \varphi^{2} & \varepsilon=-1\end{cases} \\
p=e C^{2} \frac{\varepsilon e k}{K+2 k r^{2}}, \quad \mu=k \varepsilon e \frac{3 K+2 k r^{2}}{\left(K+2 k r^{2}\right)^{2}} \quad \varepsilon= \pm 1 \quad e= \pm 1
\end{gathered}
$$

mit den willkürlichen Konstanten $k$ und $K ; \varepsilon=e=1$ liefert die von KoHLer und Chaо [2] gefundene Metrik.

\section{Strahlungsfeld - Fall 1}

Aus

$$
\begin{aligned}
\Omega_{i k} & =k_{i} k_{k}+H z_{i} z_{k} \\
T_{i k} & =e H k_{i} k_{k}
\end{aligned}
$$

folgt, daß der Weyltensor vom Typ $I I_{e}$ ist. Wenn wir noch die Maxwellgleichungen

$$
F_{; k}^{i k}=0, \quad F_{; k}^{* i k}=0
$$

berücksichtigen (vgl. hierzu KUNDT [10], [11]), so folgt, daß die Strahlenkongruenz geodätisch, hyperflächennormal und expansionsfrei ist. Auch das Vektorfeld $z_{i}$ ist hyperflächennormal, der zu ihm senkrechte Unterraum ist flach. Nehmen wir zusätzlich an, daß dieser Unterraum nicht von der durch $z_{i}=\lambda x^{1},{ }_{i}$ definierten Koordinate $x^{1}$ abhängt, so erhalten wir als eine der möglichen Lösungen

$$
\begin{aligned}
d s^{2} & =z_{1}^{2}\left(d x^{1}\right)^{2}+\left(d x^{2}\right)^{2}+2 d x^{3} d x^{4} \\
T_{i k} & =-\frac{\alpha^{\prime \prime}}{z_{1}}\left(\begin{array}{llll}
0 & 0 & 0 & 0 \\
0 & 0 & 0 & 0 \\
0 & 0 & 0 & 0 \\
0 & 0 & 0 & 1
\end{array}\right) \\
F_{i k} & =\sqrt{-\frac{\alpha^{\prime \prime}}{z_{1}}}\left(\begin{array}{cccc}
0 & 0 & 0 & -z_{1} \cos \varphi \\
0 & 0 & 0 & \sin \varphi \\
0 & 0 & 0 & 0 \\
z_{1} \cos \varphi & \sin \varphi & 0 & 0
\end{array}\right) \\
z_{1} & =A x^{2}+B+\alpha\left(x^{4}\right), \quad \varphi=\varphi\left(x^{4}\right) \quad A, B \text { Konstanten. }
\end{aligned}
$$


Auch hier hat das

\section{Strahlungsfeld - Fall 2}

$$
\begin{aligned}
\Omega_{i k} & =k_{i} z_{k}+z_{i} k_{k} \\
e T_{i k} & =-k_{i} k_{k}
\end{aligned}
$$

zugeordnete Strahlungsfeld einen Weyltensor vom Typ $I I_{e}$, und die Strahlenkongruenz ist hyperflächennormal, geodätisch und expansionsfrei; dagegen ist $z_{i}$ im allgemeinen nicht rotationsfrei. In dem zu (20) analogen Spezialfall $\left(z_{i}\right.$ hyperflächennormal, Unterraum flach und von $x^{1}$ unabhängig) hat das Feld die Gestalt

$$
\begin{aligned}
& d s^{2}=b\left(d x^{1}\right)^{2}+\left(d x^{2}\right)^{2}+2 d x^{3} d x^{4}, b=\text { const. }+2 \varepsilon x^{4}, \varepsilon= \pm 1
\end{aligned}
$$

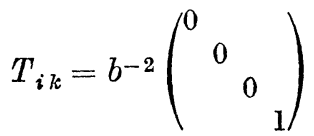

$$
\begin{aligned}
& F_{i k}=b^{-1}\left(\begin{array}{cccc}
0 & 0 & 0 & -\sqrt{b} \cos \varphi \\
0 & 0 & 0 & -\sin \varphi \\
0 & 0 & 0 & 0 \\
\sqrt{b} \cos \varphi & \sin \varphi & 0 & 0
\end{array}\right)
\end{aligned}
$$

oder

$$
\begin{aligned}
& d s^{2}=a\left(d x^{1}\right)^{2}+\left(d x^{2}\right)^{2}+2 d x^{3} d x^{4} \\
& a=\left(c_{1} x^{4}+c_{2}\right)^{2}-1
\end{aligned}
$$

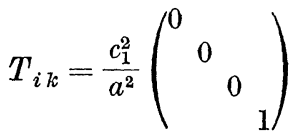

$$
\begin{aligned}
& F_{i k}=\frac{c_{1}}{a}\left(\begin{array}{cccc}
0 & 0 & 0 & -\sqrt{a} \cos \varphi \\
0 & 0 & 0 & -\sin \varphi \\
0 & 0 & 0 & 0 \\
\sqrt{a} \cos \varphi & \sin \varphi & 0 & 0
\end{array}\right)
\end{aligned}
$$

Hier sind $c_{1}$ und $c_{2}$ beliebige Konstanten, $\varphi$ ist eine beliebige Funktion von $x^{4}$. In beiden Fällen ist die fünfte Dimension zeitartig $(e=-1)$.

Herrn Prof. Dr. E. Schмutzer und allen Mitgliedern der Arbeitsgruppe Relativitätstheorie bin ich für wertvolle Diskussionen zu Dank verpflichtet.

\section{Literatur}

[1] Rosen, J.: Rev. Mod. Phys. 37, 204-214 (1965).

[2] KoHLER, M., and K. L. ChaO: Z. Naturforsch. 20a, 1537-1543 (1965).

[3] Singh, P., and S. N. Pandey: Proc. Ind. Acad. Sci. 26a, 665 (1960).

[4] Fujitani, T., M. Jkeda, and M. Matsumoto: J. Math. Kyoto Univ. 1, 43 (1961).

[5] Jkeda, M., S. Kitamura, and M. Matsumoto: J. Math. Kyoto Univ. 3, 71 (1965).

[6] Kruskal, M.: Phys. Rev. 119, 1743 (1960).

[7] SzEKERES, P.: Nuovo Cimento XLIIIA, 1062 (1966).

[8] Eisenhart, L.: Riemannian Geometry. Princeton: University Press 1926.

[9] Ehlers, J.: Abh. Mainz. Akad. Wiss. 1961, Nr. 11.

[10] Jordan, P., u. W. Kundt: Abh. Mainz. Akad. Wiss. 1961, Nr. 3.

[11] Kundt, W.: Z. Phys. 163, 77 (1961). 